Cā̃a Naxional del Niño Cátedra Ext. de Pediatría

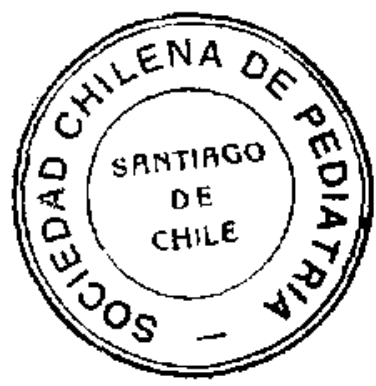

Prof. Ariztía.

\title{
TRES CASOS DE DERMATITIS SEBORREICA
}

\author{
Pror \\ JULIO SCHWARZENBERG $\mathbb{L}$. \\ Jefe de Servicio. \\ BLANCA MONTERO \\ Ayudante. \\ y EUGENIO VALLE $Q$. \\ Ayudante.
}

De entre una serie de casos de dermatitis seborreica, que hemos podido observar en nuestro Servicio, hemos seleccionado para esta presentación los tres enfermitos que nos parecen de mayot interés por la intensidad y extensión de sus lesiones cutáneas y por la gravedad de su disergia, de los trastornos nutritivos y de otras complicaciones que presentaron. En dos de los casos la dermatitis muestra todas las características de una eritrodermia descamativa (Leiner) y en el otro, hermano gemelo de uno de los anteriores, una evolución semejante, pero con caracteres mucho menos intensos en todas sus manifestaciones. En este último, la alimentación ha podido llevarse, salvo un período intermedio corto en que hubo que volver a la leche humana, sistemáticamente por el camino que istimamos ideal para la modificación flavorable de estos estados. En cambio. en los otros dos, las infecciones graves frecuentes y los trastornos nutritives paraenterales concomitantes y de gran repercusión sobre el estado general, nos obligaron muchas veces a desentendernos de la alteración constitucional y funcional cutánea, limitándonos a instituir simplemente los regímenes que aquellos exigían. Las curvas adjuntas denuestran que el progreso ponderal se hace en muy buenas condiciones en la dermatitis más leve, frente a nna evolución oscilante, arrastrada o francamente desfavorable, en las dos más graves. Las lesiones 
de la piel se han influenciado favorablemente en los tres casos bajo el uso sistemático de la pasta con sulfarilamida al $10 \%$, scbre todo cuando aparecían brotes de piodermia secundaria: pero sin que obtuviénamos una curación definitiva de las lesiones. Estimamos que las transfusiones sanguineas nos han prestado una ayuda apreciable e indiscutible tanto para combatir la disergia, los estados sépticos, como para modificar las alteraciones cutáneas.

Obserwzción N.99658. - R. A. O. La madre ha tenido 14 hijos. Este nace en parto gemelar e ingresa a la edad de 5 dias, pesando 2,440 grs. Longitud: 47 cms.; cráneo: $32 \mathrm{I} / 2$; tórax: $301 / 2$ cms. Prematuro, con ictericia leve, descamación generalizada de la piel, que se desprende en placas finas en la cara, formando en el tronco superficies extensas. separadas por surcos lineales sin eritrodermia. Higado y bazo están aumentados de volumen. Onfalitis ligera que cura con los cuidados habituales. En la primera semana de estada en el servicio aparece un eritema glúteo-genital intenso y edema de los genitales, que regresa al cabo de 10 días. En la zona que limita con la piel sana hay una descamación intensa. Al mes de edad aparecen pequeñas pápulas rojas en ambas mejillas que confluyen, formando placas ásperas al tacto. Al mismo tiempo se observan elementos aislados de seborrea en el cuero cabelludo. Días después estas lesiones se extienden a todo el tronco y se acompañan de intértrigo húmedo o simplemente eritrodérmico, que se localiza en las axilas, codos. regiones inguinales y retro-auriculares.

Con la generalización de estas lesiones se manificsta una disergia acentuada. Entre los elementos de dermatitis aparece un penfigoide, que empieza en las zonas de maceración o irritación mecánica y se extiende, evolucionando por brotes sucesivos, a tóda la superficie cutánéa. Se forman además pequeños abscesos en el cuello. Se presenta una rinofaringitis y una otitis congestiva primero y supurada después. Se inicia a esta altura una serie de transfusiones de $50 \mathrm{cc}$. cada una, llegando a un total de 10 . 'A' pesar de esto, sa mantiene un estado febril y la curva de peso se estaciona. En atención a esto se hace entonces una cura con sulfanilamida, empezando con 1,5 grs. el nrimer dia. y continuando con $0,75,0,75,0,75$, 0,625 y 0,375 grs. sucesivamente, hasta enterar en el curso de 6 días ia dosis total de 4,75 grs.

Se observa de inmediato una mejoría de la piel y del estado general dal niño, notable sobre todo en la mayor facilidad con que acepta el alimento, con lo cual se obtiene un rápido progreso en la curva ponderal. 


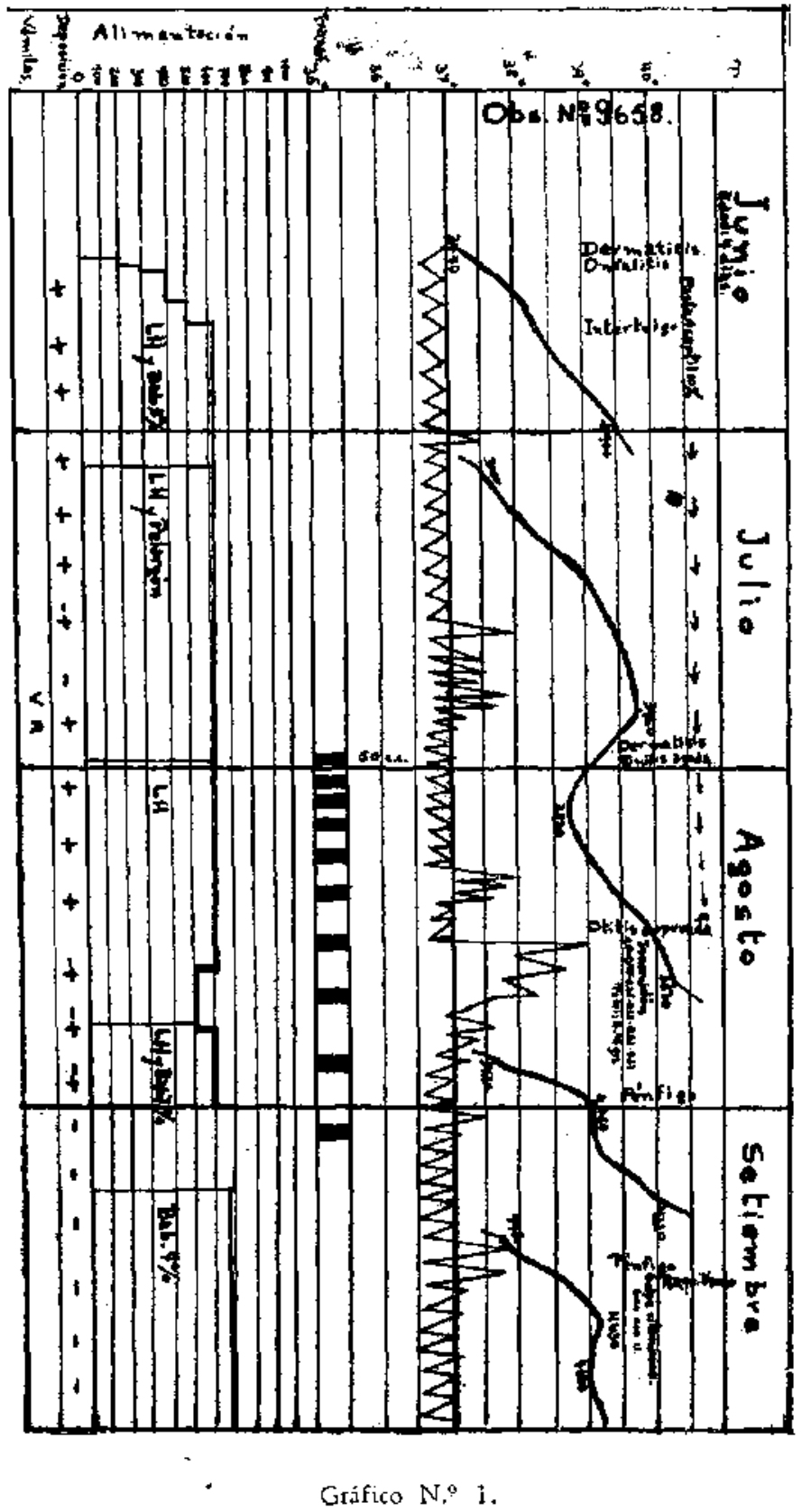


A los tres meses de edad se compraeba clínica y radiológicamente un raquitismo, que se trata con ull golpe vitaminico con Radsterin (600.000 U., o sea, 15 mmggrs. de vitamina D).

Después el niño es trasladado a otro Servicio, en el que se observan todavía por varios meses infecciones rinofaríngeas y supuraciones del oído, a veces con temperatura alta: pero que no detienen un buen progreso ponderal. Desde los cuatro meses en adelante se atenúan not́ablementz las manifestaciones cutáneas y se anotan manifestaciones muy discre tas de seborrea y en forma ocasional, intértrigo de los pliegues. Actualmente, a los 11 meses. tiene lla piel completamente sana y quedan solamente algunas pequeñas costras en el cuero cabelludo.

En la época de la generalización de sus lesiones, cuando comienzan sus infecciones y antes de iniciar las transfusiones, un hemograma da el siguiente resultado:

Glóbulos rojos 5.000.000, gl. blancos 8.400, Hgb. 106, Bas. 1. Eos. 7, Juv. 2, Bac. 8, Segm. 9. Linf. 66, Mon. 7.

Repetido dos meses despues, ya lejos de las transfusiones, nos da:

G1. r. 4.400 .000 , Gl. b. 10.400 , Hgb. $90 \%$, Eios. 2. Bac. 6, Segm. 28, Linf. 56. Mon. 8.

Epicrisis: Gemelo, que hace un buen desarrollo y tolera bien la alimentación prescrita, en el que se exacerban y generalizan las manifestaciones de una dermaticis seborreica, al parecer. bajo la influencia de infecciones cutáneas secundarias (penfigoide y abscesos) y que mejoran visiblemente con sulfanilamida per os $y$ transfusiones, a tal punto que la reaparición posterior de aquellas no vuelve a empeorar la dermaritis.

Observación N.9 9659. - L. A! O. Hermanò gemelo del anterior, ingresa como él a los 5 días de edad con un peso de 2,160 grs. Ictericia leve, descamación fina generalizada, onfalitis leve, que demora algunos días en desaparecer. Poco después eritema intenso glúteo-genital y edema de los genitales. El edema desaparece rápidamente con una pasta de prontosil al $10 \%$; pero la etitrodermia se mantiene. Al mes de edad se observa una descamación supra e interciliat, que luego se extiende al cuero cabelludo y al dorso. Después se comprueban elementos papulosos en ambas mejillas, alrededor $\mathrm{d} z$ los ojos y en el cuero cabelludo, En el curso de 10 días, toda la piel se presenta enrojecida $\mathrm{y}$ sembrada de pápulas de diverso tamaño y de color rojo obscuro. Se inicia una descamación 


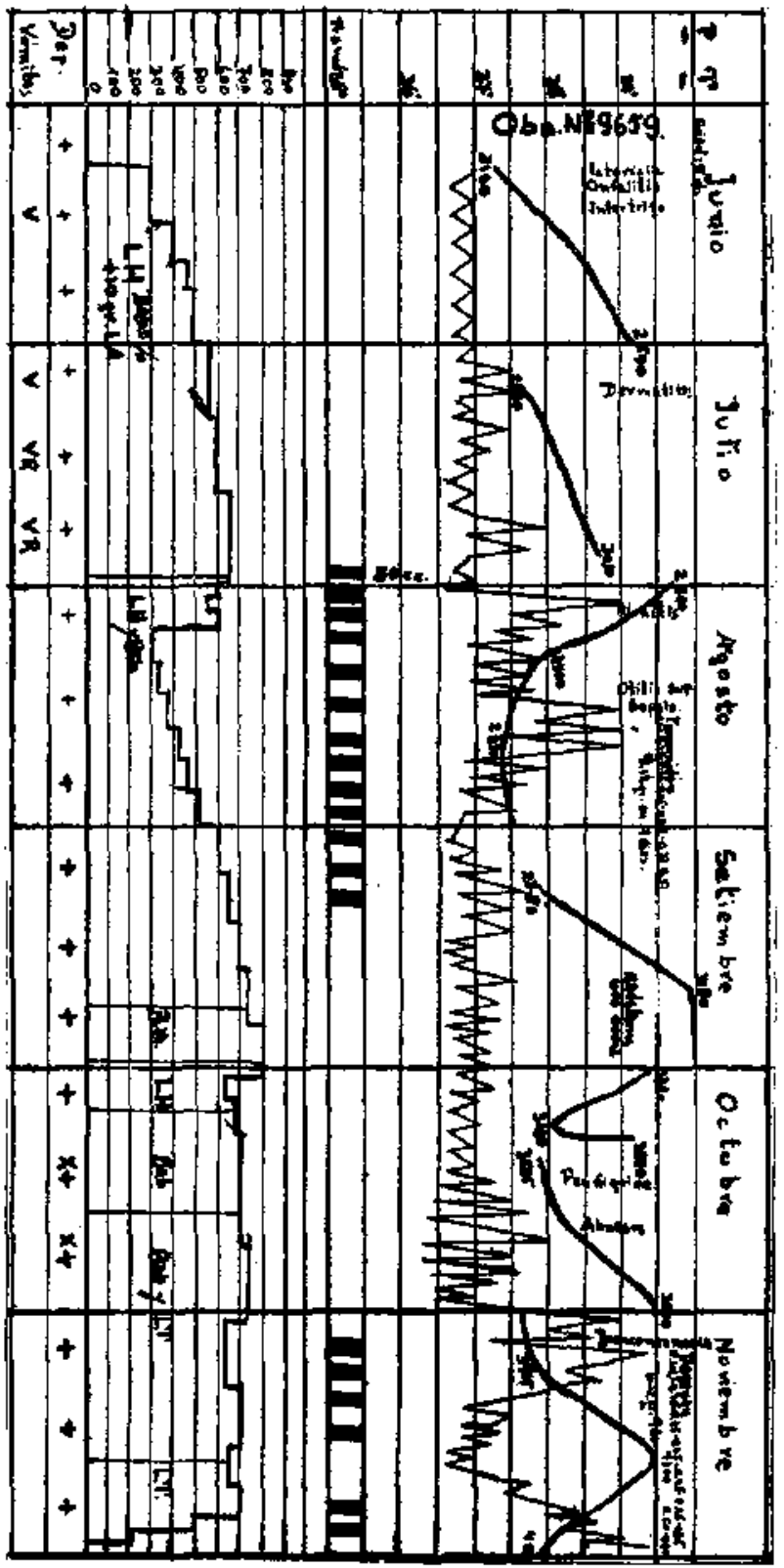

Gráfico $N^{8} 2$. 
generalizada en grandes placas. que al desprenderse de - sus bordes, dejan surcos que sangran.

Al mismo tiempo el niño hace infecciones rinofaríngeas repetiđas que producen alzas térmicas pequeñas. Desde entonces el peso se estaciona, desmejora el estado general, se alteran las deposiciones, se producen vómitos y regurgitaciones frecuentes y la alimentación se hace difícil. A pesar de un tégimen de leche humana exclusiva y de la iniciación de una serie de transfusiones ( $50 \mathrm{cc}$. cada una $y$ un total de 13), el niño se agrava. Las infecciones van de una rinitis aguda a una ctitis supurada doble y se manifiesta uria deshidratación intensa $y$ progresiva. Las fontanelas están completamente hundidas, la mucosa bucal roja y cubierta de algorra, las córneas opacas y la piel se ha tornado rojo intenso, e infiltrada con una descamación en grandes trozos. Hay cscaras de decúbito, el niño ne llora, los tonos cardiacos están apagados y la respiración es superficial. Un gran meteorisno impide la palpación abdominal. La temperatura oscila entre $38^{\circ}$ y $39^{\circ}$ y en 12 dias el nifĩe ha perdido 700 grs., casi $1 / 4$ de su peso.

En estas condiciones, además de los cambios dietéticos obligados por el trastorno nutritivo que presentaba el enfermito, y las transfusiones ya iniciadas, se hace una cura con sulfanilamida (Inmunidón) en dosis diarias de $1,5,0,75,0,75$, $0.75,0.625,0,375,0,375$ grs., alcanzando un total de 5,125 grs. Se ptoduce una cianosis intensa, que distrinuye espontámeamente al tercer dia, a pesar de la continuación del tratamiento.

Durante el segundo día del tratamiento se produjo un cuadro meningo-encef́́lico con contracturas generalizadas, opistótonos, fontanela tensa, nistagmus, anisocoria y respiración quejumbrosa. La punción lumbar da un líquido céfaloraquídeo normal; pero a presión.

Desde el día siguiente, el niño empieza a mejorar franca y progresivamente y al cumplir los tres meses de edad ha recuperado 360 grs. El estado psiquico ha cambiado mucho, sigue con la vista, se ríe y llora por su alimento. La piel conserva su coloración roja; pero está lisa, elástica y no se descama. Quedan solamente pequeñas pápulas y costras en la piel del cráneo, que presenta además una alopeía completa.

Como en el hermano, a los $31 / 2$ meses de edad se hace un golpe vitamínico con Radsterin (15 mmgrs.).

En esos días aparecen clementos pemfigoideos y pequeños abscesos y se produce un trastorno nutritivo pataenteral. que obliga a instituir una dieta hidrica y una realimentación con leche humana, con lo cu'al se obtiene una reparación tá- 
pida. Su piel se mantiene en buenas condiciones y la dermatitis no empeora.

Trasladado después de este incidente a otro Servicio, presenta a $\operatorname{los} 4$ meses nuevamente una otitis supurada, hace en seguida una bronconeumcria, de la que mejora; pero a los 51,2 meses fallece a consecuencias de una nueva bronconeumonia. a pesar de un tratamiento hecho con Dagénan y transfusiorkes sanguíneas.

Las leciones de la piel no habizin vuel to a exacerbarse $y$ se mantuvieron aún nás mitigadas que la que nosotros habíanos cbservado en e! Servicio.

En el primer hemograma, hecho artes de la primera seric de transfusiones nos da: Gl. r. 5.000.000, Gl. bl. 14.100, Hgb, $115 \%$, Eos. 7. Juv. 1, Bac. 10, Segm. 7. Linf. 65, Mon. 10.

E1 segundo, que se hizo al término de la primera cura sulfanilamidica, revela: Gl. r. 4.000.000, G1. bl, 25.400 , Hgb. 88, Eos. 7. Bac. 5. Segm. 19, Linf. 67. Mon. 2.

E! tercer hemograma, que se torio en la iniciación de la primera bronconcumonia $y$ antes de iniciar una nueva serie de transfusiones, da: Gl. r. 4.000.000?, Gl. bl. 10.000, Hgb. 85, Eos. 4, Bac. 21. Segm. 22, Linf. 45, Mor. 7.

Epicrisis: Como en el caso anterior, su hermano gemelo. manifiesta desde los primeros días lesiones de la piel yue se generalizan y agravan hacia el segundo nies de vida, aqui parece bajo la influencia de una infección paracutánea (rinitis, otitis, sepsis) y que curan también bajo un tratamiento de sulfanilamida per os y transfusiones. Contratiamente a aquél, cste niño mantien'e e intensifica infecciones graves de carácter séptico. se recupera en forma lenta y vuelve atrás ante cada una de las infecciones que interfieren durante su reparación. hasta que fallece a los $5 \frac{1}{2}$ meses por una bronconeumonia. A pesar de las nuevas infecciones y los cambios de réginen, su dermatitis no vuelve a intensificarse.

Observación N. 10015 . - V. S. C. P. Hijo de madre de 55 años, multípara de 10 , cuyo embarazo fué confundido con un misma uterino. El niño nace por la intervención quirúrgica y cufre de una asfixia pasajera: peso: 1.830 grs. En la maternidad se mantuvo con leche humana ordeñada.

Ingresa a la edad de 27 días, con un peso de $1.900 \mathrm{grs.}$; lcngitud: $47 \mathrm{cms}$; cráneo: $32, y$ tórax: $27 \mathrm{cms}$. Niño muy flaco, mal estado general, erosiones infectadas en ambos talones, intértrigo retroauricular y descamación generalizada de la piel en pequeñas placas. 


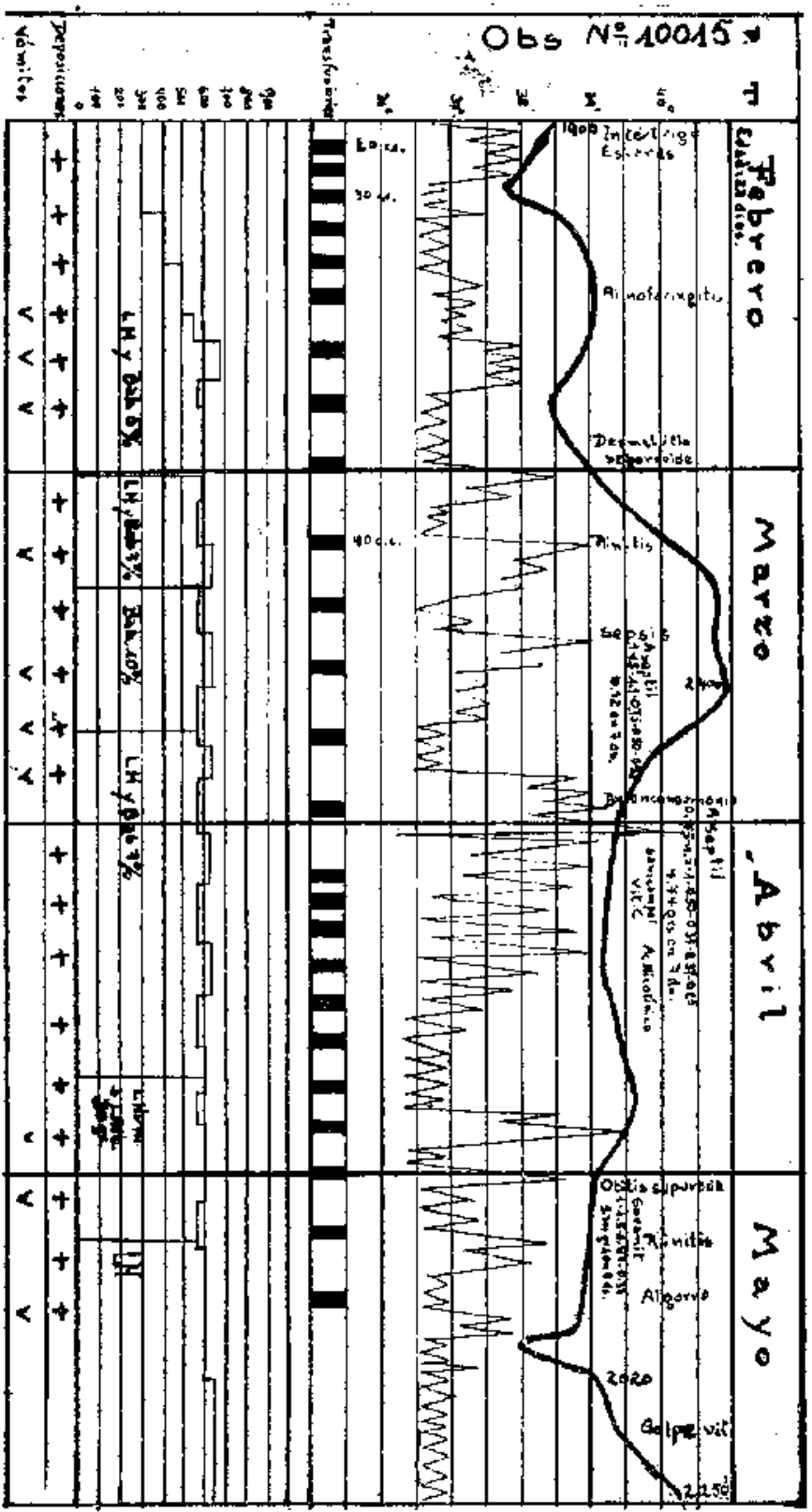

Gráfico N. 3. 
Después de unos días de estacionamiento, comienza a progresar en peso y las infecciones de la piel mejoran; pero se produce un intértrigo glúteo-genical rebelde, que se mantiene, a pesar de que se le trata con pasta de sulfanilamida al $10 \%$ durante veinte días. Al mismo tiempo, frecuentes rinofaringitis mantienen una febricula y producen trastornos nutritives agudos paraenterales sucesivos. Hacia los dos meses de edad se exacerba la dermatitis; la elpidermis se descama en grandes trczos, en los pliegues articulares se forman surcos profundos que sangran, toda la piel aparece roja e infiltrada bajo las grandes escamas, que se desprenden espontáneamiente o por el más leve roce. Durante el baño caen trozos de epidermis de varios centimetros de diámetro.

En estas condiciones la infección se exacerba. un alza de temperatura altera el estado generai y toma el carácter ya franco de una sepsis. En vista de esto, y como agregado a las transfusiones que ya se estaban haciendo, se indica un tratamiento con sulfanilamida (Aseptil), en dosis de 1,0, 1,5, 1.0 $1,28,0,75,0,50.0,50$ grs. diarios, respectivamente $\mathrm{v}$ alcanzando una dosis total de 6,53 grs. Las primeras dosis provocaron una cianosis intensa, que se fué atenuando espontáneamente. Por lo demás, no se observó ningún síntoma de intolerancia. Con el tratamiento se obtuvo una ligera mejoría del estado general, la temperatutra bajó y la piel se modificó algo.

A los pocos dias se presentan signos meníngeos que nos obligan a hacer una punción lumbar. En el liquido céfaloraquídeo se encuentra una reacción de Pandy positiva y albúmina 0,40 ; el resto del examen es normal. La temperatura vuelve a elevarse, aparecen signos de una bronconeumonía con pésimo estado general, deshidratación intensa y gran dificultad en la alimentación. Frente a la gravedad de este cuadro se vuelve a hacet un tratamiento con sulfanilamida (Asseptil), después de un descanso de 10 dias. Esta vez se admiristraron $0,75,1,5,1,0,0,50,0,37,0,25$ grs.; dosis total 4,37 grs.

Un hemograma, pedido antes de iniciarse esta cura, es informado el último dia del tratamiento y demostraba una leucocitosis de 48,000 glóbulios blancos con desviación a la izquierda y aparición de elementos jóvenes de la serie roja. Un nuevo hemograma, hecho entonces de urgencia, reveló alteraciones intensas del cuadro sanguíneo: anemia intensa, leucocitosis aún mayor e inudación de elementos jóvenes de la serie roja y blanca, como puede apreciarse por la lectura de los hemogramas. En la lámina adjunta hemos reunido algunos de los elementos característicos que se observaban en los frotes. 
5 de febrero de 1941

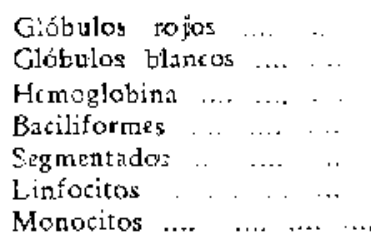

7 de abril de 1941

\begin{tabular}{|c|c|}
\hline Glóbulos & 1.900 .000 \\
\hline Glóbulos blancos & 55.000 \\
\hline Hemoglobina . . ... .. & $78 m$ \\
\hline Ecsináfilos $\quad \ldots \ldots \ldots$ & 0 \\
\hline 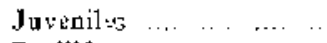 & 6 \\
\hline Baciliformes $\quad \ldots .$. & 13 \\
\hline $\begin{array}{cccc}\text { Sagmentados } & \ldots & \ldots & \ldots\end{array}$ & 20 \\
\hline I.infocitos $\ldots \ldots \ldots$ & 49 \\
\hline fonoritos .... & 10 \\
\hline
\end{tabular}

Caracteras de fó glóbalas tajos. Anisocitcis is intensa. Poiquiloxitosis. Policromatofilia mirada. Abundante punteado bacófilo $y$ metactomasia en los hemati:s. Gran cantifad de nornoblastos y megaloblastos. Prozritrobiastos basófila:

10 de abril de 1941

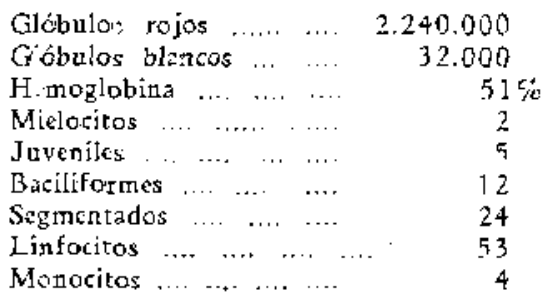

\section{7 de marzo de 1941}

Glóbulos rojos

5.000 .000

Glóbulos blancos

48.600

Himoglohina

$115 \%$

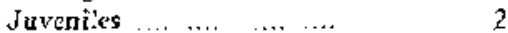

Baciliformes ...

Segmentados $\ldots . . . . \quad \ldots . . .21$

$\begin{array}{lllll}\text { Linfocitoli } & \ldots . & \ldots \ldots & \ldots & 25\end{array}$

Monocitos ...

9 de abril de 1941

Glóbulos rojos .......... 2.080 .000

Gíbulos, blancos $\ldots . . . . \quad 47.000$

Hemoglobina .........$\quad 50$

Ecsinófilos $\quad . . . \quad \ldots . . . . . \quad$ l

Basófilos .... ...

Hemocitoblatos ........

Promielocitos ........... 1

Miplecitos esonófilos ....

Mielocitos neuttófilos .... 9

Miclocitos basófilos ..... 0

Metamielocitos ... ... I?

$\begin{array}{lllll}\text { Linfoblastos } & \ldots . . & \ldots & \ldots & \end{array}$

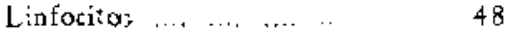

Monocitos .... ...

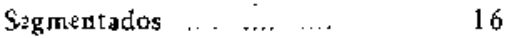

Cíulas primitivas seri:

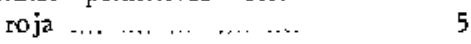

Cúracteres de loj glóbulos rojos. Anisocitosis matcada. Poiquilocitosis ligera. Whiacromasia. Abundante punte:sdo burófilo en los hematies. Numerosos reticulacitos. Arnillos de Cabot. Normoblastos y megaloblastos, metacromáticos.

14 de abril de 1941

Glóbutos rojos ..... ... 2.800 .000

G.jóbulos blanteos ... $\ldots . \quad 19.800$

Hemogloina $\ldots . . . .6 . \quad \ldots . \quad 66 \%$

$\begin{array}{llllll}\text { Miclocitos ... } & \ldots & \ldots & \ldots & \ldots & 3\end{array}$

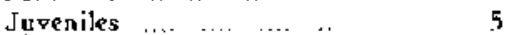

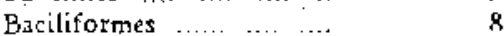

$\begin{array}{lllll}\text { Segmentidos } & \ldots & \ldots & \ldots & \ldots\end{array}$

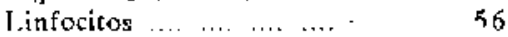

Monocitos ....... ... 
Caracteres de los glóbulos rojos. Anisocitosis menos marcada que er los eximeres anterioras: metacromaia poco intcnsa: punteado basófílo en alganos hematics. Normobiastos $y$ megaloblactos escatos. No se encuentean células primordiales de ta serie roj:.

Plaquetas abundantes, de forma y tamaño normales.

En resumen, ha habido en las úttimas 24 horas un cambio. en sentido de ser menos marcada la presentia de elementos jóvenes d: la médula y menos altetación en los hematís.

\section{1 de abril de 1941}

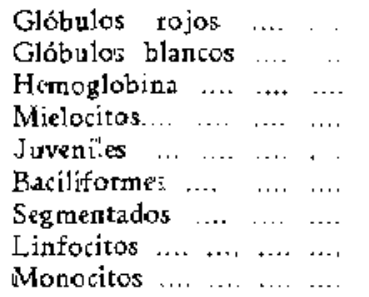

$4.500,000$

13.700

5 de mayo de 1941

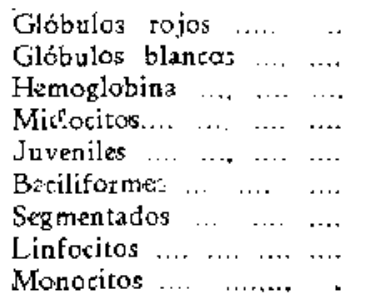

4.600 .000

19.000

$90 \%$

1
Homaties de morfologia nomal. Muy escasos normoblastọs. Colotación normal: hipocromia en alguros bemaniss. Puede con:iderare que la fótmula bemática actualmente se acerća a 1a fórmula notmal, en comparación con los exámenes practicados anteriormente. Han retrocedido las alteraciones cualitativas cbservadas anteriormente en la satia roja.

\section{8 de abril de 1941}

Glóbulos rojos ......... 4.000 .000

Glóbulos blancos .... ... 22.400

Hemoglobina $\ldots . . . . .6 . . . \quad 85 \%$

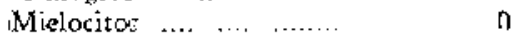

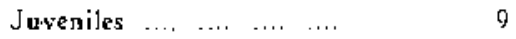

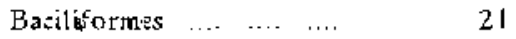

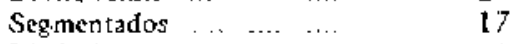

Linfocitos .... ... .... ..

Monocitos .... ... ....

\section{0 de abrill de 1941}

Glóbulos tojos ......... $\quad . \quad 4.400 .000$

Glóbulos blancos .... ... $\$ 2.000$

Hemoglobira ... .... ... $\quad 110 \%$

Valor globular ......... ... $\quad 1.25$

$\begin{array}{llllll}\text { Mieloritu' } & \ldots & \ldots & \ldots & \ldots & \ldots\end{array}$

Buciliformes $\ldots . . . . \quad \ldots . \quad 13$

Segmontados $\ldots . . . . . . \quad 36$

Linfocitos .... ...........

Monocitos ........... 2I

Escasos normoblaltos. No hay alteracicnes de los hematies. Abundartes plaquetas.

20 de mayo de 194i

Glóbulos rojos ..........

5.425 .000

Glóbnlos blancos .... ... $\quad 40.700$

Hemaglobina $\ldots$.... $\quad \ldots$

$\begin{array}{lllll}\text { Mielosir } \sigma^{-} & \ldots & \ldots & \ldots & \ldots\end{array}$

Juveniles $\ldots . . . . . \quad \ldots, \quad \ldots$...

Batififormes $\ldots . . . . .$.

Segmentados .... ... .

Linfocitos 110 n

Glábulos rojcp;

4.500 .000

Glóbulos blancos...

27.000

Hemoglobina ... .......

B-cilifermes

2

Segmentados ... .. .... 26

Linforitos ........... 64

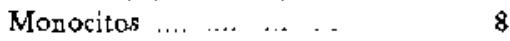

Monocitos

Promidocito's a.

Hemocitoblastos .... .. $\quad 1$ 
Degeneración nuclear en varios gra* nulocitos. Eritroblastos muy escasos.

$$
25 \text { de mayo de } 1941
$$

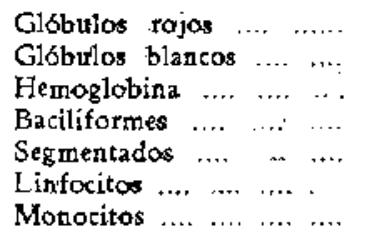

3.720 .000
23.100
$75 \%$
8
43
40
9

4 de junio de 1941

\begin{tabular}{|c|c|}
\hline Glóbulos rojos $\ldots . . . .$. & 4.200 .000 \\
\hline Glóbulos blancos & 26.000 \\
\hline Hemoglobina .... & $90 \%$ \\
\hline Baciliformes ... ........ & 4 \\
\hline$\ldots, \ldots, \quad \ldots$ & 18 \\
\hline $\begin{array}{llll}. & \ldots & \ldots & \ldots\end{array}$ & 76 \\
\hline 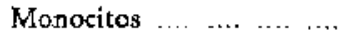 & 3 \\
\hline
\end{tabular}

Ante la gravedad del cuadro que se nos presentaba y. a pesar de la larga serie de transfusiones que ya se habia becho, se hizo una transfusión de urgencia, seguida de una nueva serie, y durante dos semanas nos ayudamos con inyecciones de Sanicampol primero, Beanemol después, ácido ascórbico intravenoso y ácido nicotínico per os. El cuadro sanguíneo mejora paulatinamente y puede considerarse casi normalizado a los 20 días después.

La piel había mejorado visiblemente, avanzando por la parte alta del tronco; la rubicundez disminuyó visiblemente y la descamación se hacia cada vez más discreta; al tacto recuperaba su elasticidad. La temperatura descendió y la curva de peso comenzaba lentamente a progresar.

15 dias después, cuando ya el niño tenía 4 meses de edad, vuelve a presentar temperaturas altas, gran alteración del estado general y baja de peso. Se comprueba una otitis supurada y una algorra intensa. Nuevamente recurrimos a la quimioterapia, esta vez con Soranil (calcio-sulfopiridina) en dosis de $1,1,5,0,87,0,37$ grs., o sea, un total de 3,64 grs. Las a1teraciones sanguineas son de menor intensidad y se acentúan, aunque en mucho menor escala que la vez anterior, solamente días después de terminado el tratamiento y despriés del descenso de la temperatura, época en la que el niño fué tomando todo el aspecto y la reacción general de un descompuesto. Sin embargo, hace algunos días, se nota una notable mejoría de su estado general, psíquis más despejado, alimentación más fácil y ascenso lento de la curva de peso. Aparece nuevamente una descamación laminar de la piel en algunas partes deí tronco conn ligera eritrodermia. La piel mejota en seguida totalmente, la curva de peso sigue ascendiendo y el estado general del niño y su psíquis alicanzaron un nivel que no habían presentado hasta la fecha. La anemia y la fórmula sanguínea se normalizan, quedando únicamente una leucocitosis alta. 
Epicrisis: Niño nacido en circunstancias anormales, que a semejanza del anterior, presenta una afección de la piel dẹ tipo de una eritrodermia descamativa (Leiner típico) de $\mathrm{ca}$ rácter serio por su extensión $e$ intensidad, como por la diser. gia grave que mantiene durante meses y por la repercusión alarmante que se manifiesta en el cuadro hemático, seguido sistemáticamente. Parece que en un principio la dermatitis se exacerba en este caso bajo la influencia de piodermias; pero una vez que éstas curacon, se manifiesta daramente la acción de las infecciones paracutáneas (rinitis, otitis, sepsis, etc.). Los hemogramas demuestran la evolución sombría de los cuadros hemáticos y a pesar de ello, en la actualidad el enfermito está recuperándose francamente.

Resumiendo, podemos establecer que se trata de tres n1ños prematuros, hijos de madres multíparas, de edad avanzada, que presentan desde los primeros días de la vida una dermatitis seborreica, que se intensifica hacia el segundo mes, bajo la influencia aparente de las infecciones cutáneas y paracutáneas, que interfieren permanentemente en el desarrollo de ellos, como manifestación de su disergía acentuada y como provocadoras de trastornos nutritivos continuos y graves. Aunque la evolución general se hace en cada uno de ellos bajo modalidades diferentes, estas no son más que la expresión de la gradación diversa de una misma alteración otgánica y funcional. La intención de llegar al régimen adecuado para la modificación de la constitución y de la diátesis $y$ de mantenerlo durante el tiempo necesario, está subordinada a los accidentes intercurrentes que se presentan y se ve tanto más entorpecida, cuanto mayor sea desde un principio el compro. miso del estado general. Y así vemos, que reacciona en forma muy farorable el primero y más leve de nuestros casos, qu presenta problemas dietéticos y terapéuticos de solución relativamente fácil, mientras que en los otros dos son de tal mul. tiplicidad y gravedad, que es materialmente imposible atenerse en esas condiciones, a una línea de conducta preestablecida. A pesar de todos los esfuerzos hechos, uno de los últimos fallece altededor de los 6 meses de edad y a pesar de haber mejorado de su afección cutánea; mientras que el otro so. brevive, después de haber pasado por un estado de predescomposición, cuya reparación actual nos alienta para esperar su salvación definitiva, pese a las profundas alteraciones hematológicas que presentó en el trascurso de sus infecciones.

Interesante nos parece establecer cuál puede haber sido la causa más probable de las alteraciones sanguíneas tan profun- 
das, que presentó el último de estos casos. A primera vista podría haberse pensado en una acción tóxica provocada por la sulfanilamida; pero los hemogramas no presentan las caracteristicas que se señalan como específicas para estas circunstancias. Llama la atención. en seguida, que la leucocitosis elevada $(48,000$ glóbulos blancos) y una fuerte desviación a la izquierda con presencia de elementos juveniles de la serie blanca, se presenta ya antes de la iniciación del tratamiento quimicterápico y cuando la infección estaba ya en pleno auge. Cierto es que Ja anemia se intensifica y que los elementos tóvenes, tanto de ta serie roja como blanca, van en aumento durante el trascurso del tratamiento y que todas estas alteracio. nes regresan paulatinamente después de la suspensión de él. hasta obtenerse un hemograma muy cercano a la normal en los días en que el niño vuelve a subir de peso y en los que la temperatura se había hecho normal. Pero una nueva reinfección, si bien aumenta la leucocitosis $(22.400$ glóbulos blancos), tratada una $\mathrm{Vez}$ más con sulfopiridina, no da durante este tratamiento mảs que una leucocitosis moderada (19.000 gl. bl.) y sólo un mielocito dentro de una desviación a la iz. quierda intensa, sin repercusión alguna sobre el cuadro rojo, ni en cantidad ni en calidad. Recalcamos que hasta esa altura esta fase infécciosa había influenciado muy poco, en todo caso, mucho menos que la anterior, el estado general y las condiciones del niño. En cambio, ya muchos dias después de esta quimicterapia, cuando el enfermo estaba aún en plena sepsis y entraba en un perícdo de franca descomposición, el hemograma nos presenta nuevamente lina alteración semejante a la primera vez; pero mucho menos intensa, y que salvo una leucocitosis todavía alta, se normaliza junto con la acentuación de la mejoría del estado general, como puede observarse en la curva y por los hemogramas.

Por estas razones nos inclinamos a creer que este cuadro hemático tan grave, nada tiene que ver con la quimioterapia y que es. en cambio, la consecuencia de una sepsis gravísima, lia primera vez, y más leve en la segunda etapa, en un prenaturc cuya labilidad hematopoyética, no puede extrañar. Abonan es. te parecer. la gravedad de las alteraciones y su menor intensidad en la primera y en la segunda infección, respectivamente, y su independencia del tratamiento, sobre todo, la última vez. Por lo demás, hemos observado cuadros hemáticos semejantes, aunque no de esta intensidad, en lactantes y prematuros infectados, que no ban sido sometidos a esta quimioterapia. 